\title{
The Effects of a Combination of 3D Virtual Reality and Hands-on Horticultural Activities on Community-dwelling Older Adults' Mental Health: A Quasi-Experimental Study
}

\section{Ching-Chih Fan}

En Chu Kong Hospital

Cheuk-Sing Choy

Yuanpei University of Medical Technology

Chiu-Mieh Huang

National Yang Ming Chiao Tung University

Po-Sheng Chih

En Chu Kong Hospital

Chia-Chiang Lee

National Taiwan Normal University

Fen-He Lin

National Yang Ming Chiao Tung University

Jong-Long Guo ( $\nabla$ jonglong@ntnu.edu.tw)

National Taiwan Normal University

\section{Research Article}

Keywords: 3D virtual reality, Horticultural therapy, Community-dwelling, Older adults, Mental health

Posted Date: December 13th, 2021

DOI: https://doi.org/10.21203/rs.3.rs-948202/v1

License: (c) (1) This work is licensed under a Creative Commons Attribution 4.0 International License. Read Full License 


\section{Abstract}

Background: Aging societies are of public health concern worldwide. It is critical to develop strategies that harness technology to enhance older adults' mental health.

Methods: This study aimed to explore the effects of a combination of 3D virtual reality (VR) and handson horticultural activities on the mental health of community-dwelling older adults. The study used a quasi-experimental design. A total of 62 community-dwelling older adults were recruited and assigned to the experimental $(n=32)$ and comparison groups $(n=30)$. The members of the experimental group participated in an 8-week intervention program. Participants of both groups completed before-and-after intervention measurements for outcome variables that included perceived self-esteem, depression, isolation, mastery and achievement motives, which were analyzed using the generalized estimating equation (GEE).

Results: GEE analyses indicated that the experimental group showed significant post intervention improvements in scores for self-esteem $(\beta=1.66, P=.015)$, isolation $(\beta=-0.96, P=.008)$, mastery $(\beta=1.04$, $P=.042)$, and achievement motives $(\beta=1.30, P=.034)$ compared to the control group.

Conclusions: This study found beneficial effects of a combination of three-dimensional virtual reality and hands-on horticultural activities on community-dwelling older adults' mental health. These findings suggest that future implementations of this program on a large scale could improve the mental health of older adults.

Trial Registration: The study was posted in the www.clinicaltrials.gov (NCT05087654) on 21/10/2021. It was approved by the Institutional Review Board of En Chu Kong Hospital and performed in accordance with the Declaration of Helsinki.

\section{Background}

Advances in healthcare technology have extended the average life expectancy of humans. The aging of populations has, therefore, become a global public health issue. Older adults typically face a decline in their physical health in the final stage of their lives, and often feel depressed and lonely due to the lack of interpersonal interaction after their retirement [1]. Previous studies have tended to focus on strategies for the promotion of physiological health, such as strengthening muscles and improving exercise and nutrition. As a result, there are very few studies on the promotion of mental health [2]. Of the few studies that do address mental health, a large-scale survey indicated that $43 \%$ of people aged over 60 felt lonely [3]. Another study investigated 2,288 community residents aged $55-85$, and found a significant correlation between depression and loneliness, self-esteem, and mastery after 6 years of follow-up [4]. A systematic review found a significant negative correlation between self-esteem and depression [5]. Further, the importance of older adults' mental health on their physical wellbeing has also been recently recognized. A 6-year population-based cohort study concluded that older adults' mastery attenuated the adverse effect of frailty on decline in physical function [6]. Strategies to improve the mental health of the 
elderly have recently attracted research attention, and work often focuses on quantifying the potential role of various forms of therapy.

Horticultural therapy is known to stimulate sensory functions, enabling the elderly to achieve satisfaction and improve their self-esteem $[7,8]$. Gardening activities often involve substantial interaction with peers, resulting in increased opportunities for social interaction and reduced depression and loneliness $[9,10]$. In addition to this, participants also get a sense of achievement [11]. A systematic review found that gardening activities can promote overall health and quality of life, physical strength, fitness and flexibility, cognitive ability, and socialization [8, 12]. Besides depression and loneliness, other critical variables associated with high mental health such as self-esteem, mastery, and achievement motives are still not studied.

As a result of advancing information and communication technology, virtual reality (VR) builds a virtual world with three-dimensional (3D) simulation. It can provide a virtual learning environment and can create stereo sound effects. 3D VR simulations provide an "immersive experience" that enables people to interact with virtual scenes and improve intervention effects. It has been widely used in healthcare for purposes of rehabilitation [13], pain management [14], cognitive training [15], and improvements of body function [16, 17]. A past study indicated that older adults have a high acceptance of VR intervention [18]. For example, 30 older adults who underwent a 6-week VR program agreed that VR experiences were useful, easy to use, and pleasant [18]. Thus, 3D VR shows promise in improving older adults' physical and mental health. Moreover, older adults were interested in digital games that can connect with others, and provide feedback or rewards which can increase their motivation [19].

A prior study found that the experimental group showed significantly improved health status, perceived meaning in life, perceived mattering, and significantly reduced depression and loneliness compared to those of the control group immediately after the intervention; these effects persisted for up to 2 months [20]. Another study revealed that a combination of 3D VR and hands-on aromatherapy significantly improved institutionalized older adults' happiness, perceived stress, sleep quality, meditation experience, and life satisfaction [21]. VR intervention improves the learners' academic performance [22, 23]. The experience and effect of participation are critical factors that contribute to future continuous use by older adults [24]. However, this type of study design has not been applied in populations of communitydwelling older adults. Thus, this study aimed to explore the effects of a combination of 3D VR and handson horticultural activities on community-dwelling older adults' mental health. This study specifically examines community-dwelling older adults in Taiwan, whose population is officially an aged population, as of 2018.

\section{Methods}

\section{Participants}

The study adopted a quasi-experimental design and was approved from the Research Ethics Review Committee of En Chu Kong Hospital (ECKIRB1090503). The study was conducted in two community 
long-term care facilities in New Taipei City and were designated one facility as an experimental and the other as comparison group. Participants were recruited via posters and verbal advertisements made by the staff during internal activities. The selection criteria included intact cognition, the ability to understand verbal instruction and the ability to operate a VR joystick independently. The exclusion criteria included a history of hand dysfunction, severe visual and hearing impairment, being allergic to plants or pollen, and a current illness such as epilepsy or stroke.

\section{Sample estimation}

G*power 3.1 (HHU; Germany) software [25] was used to estimate sample size using an effect size of 0.35, an a error probability significant level set to 0.05 and power set to 0.8 ; we found that a sample size of 52 was adequate. After considering $20 \%$ attrition rate, we decided to recruit 62 participants, 32 and 30 in the experimental and comparison group, respectively.

\section{Participants' Enrollment and Assessment}

The enrollment and assessment process is shown in Figure 1. After recruiting the two long-term care facilities, a research associate approached the executive director and staff to explain the purpose of the study and research procedure. After obtaining the administration's consent, we distributed recruiting messages to potential participants and explained the informed consent form face-to-face. Each participant completed the consent form before the collection of baseline data, which was done one-byone in a quiet and independent room at the facility. During the study period, 1 horticultural therapist, 2 VR operation instructors and 4 staff of station were present to ensure the smooth progress of the study. The participants of the comparison group received scheduled activities, such as physical fitness, paper cutting, etc., without any gardening activities during the intervention and follow-up period.

\section{The Combination of 3D VR and Hands-on Horticultural Activities}

The intervention program consisted of 8 two-hour sessions that were conducted once a week for 8 consecutive weeks. Before the intervention implementation, research staff advised the participants on how to wear the VR helmet, operate the VR joystick and familiarized them with the VR scenes. The participants of the experimental group were divided into 4 groups during the weekly activities, and each group was assisted by a facility staff. Before the activity, staff explained the theme of the week and performed the hands-on horticultural activity after the VR operation.

\section{Measurement Instruments}

Sociodemographic variables assessed at baseline included age, sex, and educational level.

\section{Rosenberg Self-Esteem Scale}

This scale has been used previously in a study targeting older adults [26], and has good reliability and validity [27]. The scale consists of 14 items and each item is scored on a Likert-type scale that ranges 
from 1 (strongly agree) to 4 (strongly disagree), with higher scores indicating a higher level of selfesteem. A sample item is "Sometimes I don't think I have any merit at all." The Cronbach's a coefficients were 0.77 and 0.89 at baseline and post-test in this study.

\section{Geriatric Depression Scale-Short Form (GDS-15)}

The Chinese version of the Geriatric Depression Scale [28] was used, which has a total of 15 items. All items were answered as either yes (1) or no (0). The total raw scores ranged from 0 to 15 , with a higher score indicating a higher level of depression. Items $1,5,7,11,13$ were reverse scored because they are of a positive nature. The same Chinese version of this scale was used to study depression and its correlates among 195 older adults living in southern rural communities in Taiwan with a Cronbach's a of 0.82 [29]. The Cronbach's a coefficients were 0.82 and 0.77 at baseline and post-test in this study.

\section{Perceived Isolation}

A short-form scale of perceived isolation was used to measure isolation. It consisted of 3 items as follows: 1. In general, how often do you feel that you lack companionship? 2. In general, how often do you feel left out? 3. In general, how often do you feel isolated from others? Each item was scored on a Likert-type scale from 1 (never) to 4 (often), with higher scores indicating a higher level of perceived isolation. The Cronbach's a of the scale during its development was 0.70 [30]. The Cronbach's a coefficients were 0.81 and 0.86 at baseline and post-test in this study.

\section{Perceived Mastery}

The Chinese version of the Perceived Mastery Scale [31] with a total of 7 items was used. Each item was scored on a Likert-type scale from 1 (strongly agree) to 4 (strongly disagree). The total raw score ranged from 7 to 28 , with a higher score indicating a higher level of perceived mastery. A previous study indicated the Cronbach's a coefficients were 0.82 [32]. The Cronbach's a coefficients were 0.78 and 0.82 at baseline and post-test in this study.

\section{Achievement Motives Scale (AMS)}

The AMS consists of two kinds of motives, referred to as Motive to Achieve Success, (MAS) and Motive to Avoid Failure (MAF) [33]. A short-form scale with 10 items was used to measure achievement motives, with 5 items each for MAS and MAF. Each item was scored on a Likert-type scale from 1 (strongly agree) to 4 (strongly disagree). A previous study indicated the Cronbach's a coefficients were 0.88 and 0.86 at MAS and MAF, respectively [34]. The Cronbach's a coefficients were 0.79 and 0.82 at MAS and MAF at baseline and post-test in this study.

\section{Horticultural Knowledge}

To examine learned horticultural knowledge, we designed a post-session evaluation with 2 questions according to the learning objectives and contents of each session. The program consisted of 8 sessions, 
thus, participants completed 16 knowledge questions.

\section{Data Analyses}

Descriptive analyses were conducted for demographic and outcome variables. A 2-tailed $t$ test and $\chi^{2}$ test were used to compare differences in age, sex, education level, and chronic disease history between the experimental and comparison groups. A generalized estimating equation (GEE) was used to investigate the effect of time point, group, and their interaction on the outcome variables. GEE analyses enable an understanding on the patterns of change and their effects at both the individual and group levels [35]. Statistical analyses were conducted using SPSS (version 23.0; IBM Corp).

\section{Results}

\section{User Statistics}

The participants' average age was 70.94 (SD 5.0) years and 69.83 (SD 3.8) years in the experimental and comparison groups, respectively. There were no statistically significant differences in participants' age, sex, education level, and chronic disease history between the experimental and comparison groups.

\section{Improvement of Outcome Variables}

The baseline scores for 5 outcome variables were not statistically significant between experimental and comparison groups (Figure 2). GEE analyses indicated that the experimental group showed significant post-intervention improvements relative to the comparison group for scores of self-esteem $(\beta=1.66, P=$ .015), isolation ( $\beta=-0.96, P=.008)$, mastery $(\beta=1.04, P=.042)$, and achievement motives $(\beta=1.30$, $P=.034)$ (Table 1). 
Table 1

Results of generalized estimating equation analyses on the five outcome variables.

GEE $^{\mathrm{a}}$ coefficient $(\beta) \quad$ SE $\quad$ Wald $\chi^{2} \quad P$-value

\section{Self-esteem}

Group (experimental group) ${ }^{b}$

0.54

$0.83 \quad 0.42$

0.519

Time (posttest) ${ }^{c}$

0.90

0.41

4.77

0.029

Group (experimental group) X time (posttest) ${ }^{d} \quad 1.66$

$0.68 \quad 5.90$

0.015

\section{Depression}

Group (experimental group) ${ }^{b}$

$-1.20$

0.82

2.14

0.143

Time (posttest) ${ }^{\mathrm{c}}$

$-0.92$

$0.41 \quad 5.00$

0.025

Group (experimental group) $X$ time (posttest) ${ }^{d}$

0.02

$0.49 \quad 0.00$

0.968

\section{Isolation}

Group (experimental group) ${ }^{b}$

0.04

$0.53 \quad 0.01$

0.943

Time (posttest) ${ }^{c}$

0.07

0.26

0.72

0.789

Group (experimental group) X time (posttest) ${ }^{d}$

$-0.96$

$0.36 \quad 7.06$

0.008

\section{Mastery}

Group (experimental group) ${ }^{b}$

0.21

0.74

0.78

0.781

Time (posttest) ${ }^{c}$

$-0.29$

$0.46 \quad 0.39$

0.534

Group (experimental group) X time (posttest) ${ }^{d}$

1.04

0.51

4.12

0.042

\section{Achievement Motives}

Group (experimental group) ${ }^{b}$

0.90

$0.84 \quad 1.15$

0.283

Time (posttest) ${ }^{c}$

0.13

$0.48 \quad 0.08$

0.779

a GEE: generalized estimating equation.

${ }^{\mathrm{b}}$ Reference group (group): comparison group.

${ }^{\mathrm{c}}$ Reference group (time): pretest.

${ }^{\mathrm{d}}$ Reference group (group time): comparison group pretest. 


\section{GEE ${ }^{a}$ coefficient $(\beta) \quad$ SE $\quad$ Wald $\chi^{2} \quad P$-value}

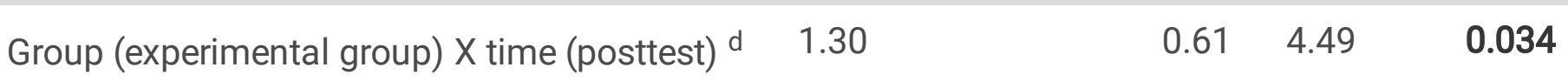

a GEE: generalized estimating equation.

${ }^{\mathrm{b}}$ Reference group (group): comparison group.

${ }^{\mathrm{c}}$ Reference group (time): pretest.

${ }^{d}$ Reference group (group time): comparison group pretest.

3D VR was very successful in the participant learning of horticultural knowledge. Only 1 participant answered a question wrong in the post-session test of the 1st and 2nd week, respectively. However, the correct answer rate of the 2-question post-session test among participants was $100 \%$ from the 3rd week, and the overall correct answer rate was as high as $99.6 \%$.

\section{Discussion}

Previous studies aimed to verify the impact of horticultural activities on the mental health of older adults. In this study, we further combined 3D VR and hands-on horticultural activities to expand the intervention program, which is in line with the e-health trend. We found that this may promote the elderly's mental health and help them successfully obtain horticultural knowledge. The positive impact of horticultural activities on the mental health of the elderly has also been supported by previous studies. For example, study participants' self-esteem was significantly improved on operating community farms $(P<.001)$ [36]. Further, most participants gave the feedback that they would like to be in contact with nature, which may improve their sense of accomplishment, relieve their stress, and improve their interpersonal interactions [36]. A previous study found that participants who used VR interactive scenarios reported improved perceived health and overall wellbeing, and reduced depression and social isolation relative to those in the comparison group (that only watched TV) [37]. Another study found that VR provides interactive learning and contributes to knowledge retention [38]. These findings are consistent with that of this study.

The intervention did not improve the perceived depression of participants, which is inconsistent with previous studies. Researchers who conducted a 10-week indoor horticultural treatment activity for 10 elderly people (average age of 75.3 years) to evaluate the intervention effectiveness of depression and loneliness, found that both had significantly improved $(P<$.001) [11]. A 3-year longitudinal study found that community-dwelling older adults who participated in domestic/gardening activities had a lower incidence of depression [39]. The intervention in this study may not be significant because the comparison group also participated in scheduled activities simultaneously, that improved perceived depression. Thus, even if the change in pre- and post-test on depression scores in the experimental group was significant $(P=.004)$, the GEE results compared with the comparison group were not significant. 
Moreover, if any participant's score in the GDS-15 (Chinese version) was less than 5 [40], it indicated the participant was not depressed. The pre-test depression score in the experimental participants was only 2.88 and they are more active compared to their institutionalized counterparts, so the improvement due to the intervention is relatively limited.

Interestingly, a previous study indicated that people with low depression have relatively high mastery [41], which is consistent with our findings. The experimental design may be the reason the post-test mastery score of the experimental participants was superior to those in the comparison group. When participants used the 3D VR or conducted horticultural activities, our research team prepared various virtual and physical plants. Each participant could independently select virtual plants to experience and then use the physical plants to make designed products. The horticultural therapist and staff guided and assisted participants in finishing their activities and products. The participants presented their horticultural products to each other and shared their feelings. We suggest that future research should adopt similar designs using horticultural activities that allow the older adults to independently select and operate horticultural material, and to share their work after completion to strengthen their perception of finishing the designed activities, to further improve perceived mastery.

Independent use of 3D VR could also promote the mental health of the elderly. For example, a previous study using a 2-week VR interactive system revealed that the participants showed a lower degree of depression and social isolation [37]. In this study, the participants in the experimental group intensively interacted with avatars to complete their gardening activities during the 3D VR scenarios, but also interacted with other participants, horticultural therapists, and staff for performing hand-on horticultural activities. Therefore, the participants in the experimental group improved significantly, which was consistent with the above research finding.

Another feature of this study is that when the participants in the experimental group experienced 3D VR horticultural activities and completed the activity, they obtained 2 virtual gold coins for each task. This feedback design enabled the participants to strive to complete the task to obtain rewards. In addition, we invited 4 older adults ( 2 males and 2 females) to examine the users' experience of VR horticultural activities before the design of study to confirm its feasibility for community-dwelling older adults. A review study indicated that low game complexity can enable older adults to play with increased motivation [19]. A previous study examined 10 community-dwelling older adults without previous use experience of VR in a semi-structured interview and 2 subsequent focus group sessions about their perceptions about using VR devices. It was found that VR is feasible to use by older adults, even for those who are immobile or reside within a care facility [42]. Our study also revealed that community-dwelling older adults could successfully complete an 8-week VR horticultural program after practicing with the assistance of the research team. In a survey that explored the use and acceptance of VR by older adults, $64.4 \%$ among them expressed that VR was easy to use, $77.8 \%$ indicated VR helped improve their mood and maintained motivation for daily activities, $80 \%$ reported that VR was very interesting, and $71.7 \%$ planned to continue to use VR [18]. The findings indicated that 3D VR could be a useful technological tool to promote the mental health of older adults. 
The intervention sites of this study are community long-term care facilities. Our findings might not generalized to frail adults in other long-term care facilities. In addition, due to the COVID-19 epidemic, all personnel strictly complied with epidemic prevention approaches in the intervention period that included wearing masks in each session. Most participants reported that the sense of smell and taste in the hands-on horticultural activities were affected by wearing masks, which may influence the effectiveness of intervention. Researchers who applied 3D VR and hand-on horticultural activities for 8-weeks to improve the mental health of institutionalized residents found that there was retention for 2 months [20]. Future studies with similar intervention programs should incorporate a longitudinal study design to explore the middle-term or long-term effects of these interventions on older adults' mental health.

\section{Conclusion}

The study found beneficial effects of a combination of 3D VR and hands-on horticultural activities on community-dwelling older adults' mental health. The findings suggest that the future successful implementation of the program on a large scale could improve the mental health of older adults.

\section{Abbreviations}

3D VR: Three-dimensional virtual reality; GEE: generalized estimating equation; GDS-15: Geriatric depression scale-short form; AMS: Achievement motives scale; MAF: Motive to avoid failure

\section{Declarations}

\section{Acknowledgments}

We would like to express our gratitude to the participants and the care facilities. This article was subsidized by the National Taiwan Normal University, Taiwan, ROC.

\section{Authors' contributions}

CCF, CSC \& JLG were responsible for the study conception and design. CCF, CSC, CMH, PSC, CCL and FHL provided administrative, technical and material support. CMH, PSC, CCL, FHL, \& GJL performed data management and statistical analysis. All authors were responsible for the drafting of the manuscript. All authors have read and agreed to the published version of the manuscript.

\section{Funding}

This research was supported by the En Chu Kong Hospital.

\section{Availability of Data and Materials}

The data used to support the findings of this study are included within the article. 


\section{Ethics Approval and Consent to Participate}

The study was posted in the www.clinicaltrials.gov (NCT05087654) on 21/10/2021, approved by the Institutional Review Board of En Chu Kong Hospital and performed in accordance with the Declaration of Helsinki. All participants provided the informed consents.

\section{Consent for publication}

Not applicable.

\section{Competing Interests}

The authors declare that they have no competing interests.

\section{References}

1. Segel-Karpas D, Ayalon L, Lachman ME. Loneliness and depressive symptoms: the moderating role of the transition into retirement. Aging Ment Health. 2018;22(1):135-140. PMID: 27624519.

2. Liu X, Ng DH-M, Seah JW-T, Munro YL, Wee S-L. Update on interventions to prevent or reduce frailty in community-dwelling older adults: a scoping review and community translation. Curr Geriatr Rep. 2019;8(2):72-86. doi: 10.1007/s13670-019-0277-1

3. Perissinotto CM, Stijacic Cenzer I, Covinsky KE. Loneliness in older persons: a predictor of functional decline and death. Arch Intern Med. 2012;172(14):1078-1083. PMID: 22710744

4. Bisschop MI, Kriegsman DM, Beekman AT, Deeg DJ. Chronic diseases and depression: the modifying role of psychosocial resources. Soc Sci Med. 2004;59(4):721-733. PMID: 15177830.

5. Sowislo JF, Orth U. Does low self-esteem predict depression and anxiety? A meta-analysis of longitudinal studies. Psychol Bull. 2013;139(1):213-240. doi: 10.1037/a0028931.

6. Lee WJ, Chen LK, Peng LN, Chiou ST, Chou P. Personal mastery attenuates the adverse effect of frailty on declines in physical function of older people: A 6-year population-based cohort study. Medicine. 2016;95(34):e4661. PMID: 27559969

7. Sullivan ME. Horticultural therapy--the role gardening plays in healing. J Am Health Care Assoc. 1979;5(3):3-8. PMID: 10316809

8. Wang D, MacMillan T. The benefits of gardening for older adults: a systematic review of the literature. Act Adapt Aging. 2013;37(2):153-181. doi: 10.1080/01924788.2013.784942.

9. Tse MMY. Therapeutic effects of an indoor gardening programme for older people living in nursing homes. J Clin Nurs. 2010;19(7-8):949-958. doi: 10.1111/j.1365-2702.2009.02803.x.

10. Masuya J, Ota K, Mashida Y. The effect of a horticultural activities program for the community elderly. Int J Nurs Clin Pract. 2017;4:266. doi: 10.15344/2394-4978/2017/266

11. Chen Y-M, Ji J-Y. Effects of horticultural therapy on psychosocial health in older nursing home residents: a preliminary study. J Nurs Res. 2015;23(3):167-171. PMID: 00134372-201509000-00003. 
12. Nicholas SO, Giang AT, Yap PLK. The effectiveness of horticultural therapy on older adults: a systematic review. J Am Med Dir Assoc. 2019;20(10):1351.e1-.e11. doi: https://doi.org/10.1016/j.jamda.2019.06.021.

13. Dermody $G$, Whitehead $L$, Wilson $G$, Glass $C$. The role of virtual reality in improving health outcomes for community-dwelling older adults: systematic review. J Med Internet Res. 2020;22(6):e17331. PMID: 32478662.

14. Benham S, Kang M, Grampurohit N. Immersive virtual reality for the management of pain in community-dwelling older adults. OTJR. 2019;39(2):90-96. PMID: 30595096.

15. White PJ, Moussavi Z. Neurocognitive treatment for a patient with Alzheimer's disease using a virtual reality navigational environment. J Exp Neurosci. 2016;10:129-135. PMID: 27840579.

16. Li Z, Han XG, Sheng J, Ma SJ. Virtual reality for improving balance in patients after stroke: A systematic review and meta-analysis. Clin Rehabil. 2016;30(5):432-440. PMID: 26141808.

17. Huang Q, Wu W, Chen X, Wu B, Wu L, Huang X, et al. Evaluating the effect and mechanism of upper limb motor function recovery induced by immersive virtual-reality-based rehabilitation for subacute stroke subjects: study protocol for a randomized controlled trial. Trials. 2019;20(1):104. PMID: 30728055.

18. Syed-Abdul S, Malwade S, Nursetyo AA, Sood M, Bhatia M, Barsasella D, et al. Virtual reality among the elderly: a usefulness and acceptance study from Taiwan. BMC Geriatr. 2019;19(1):223. PMID: 31426766.

19. Cota TT, Ishitani L. Motivation and benefits of digital games for the elderly: a systematic literature review. RBCA. 2015;7(1):2-16. doi: 10.5335/rbca.2015.4190

20. Lin TY, Huang CM, Hsu HP, Liao JY, Cheng VY, Wang SW, et al. Effects of a combination of threedimensional virtual reality and hands-on horticultural therapy on institutionalized older adults' physical and mental health: quasi-experimental design. J Med Internet Res. 2020;22(11):e19002. PMID: 33135666

21. Cheng VY, Huang CM, Liao JY, Hsu HP, Wang SW, Huang SF, et al. Combination of 3-dimensional virtual reality and hands-on aromatherapy in improving institutionalized older adults' psychological health: quasi-experimental study. J Med Internet Res. 2020;22(7):e17096. PMID: 32706660.

22. Chang C-Y, Sung H-Y, Guo J-L, Chang B-Y, Kuo F-R. Effects of spherical video-based virtual reality on nursing students' learning performance in childbirth education training. Interact Learn Environ. 2019:1-17. doi: 10.1080/10494820.2019.1661854.

23. Chen FQ, Leng YF, Ge JF, Wang DW, Li C, Chen B, et al. Effectiveness of virtual reality in nursing education: meta-analysis. J Med Internet Res. 2020;22(9):e18290. PMID: 32930664.

24. Yeh TM, Pai FY, Jeng MY. The factors affecting older adults' intention toward ongoing participation in virtual reality leisure activities. Int J Environ Res Public Health. 2019;16(3). PMID: 30691062.

25. Faul F, Erdfelder E, Buchner A, Lang AG. Statistical power analyses using G*Power 3.1: tests for correlation and regression analyses. Behav Res Methods. 2009;41(4):1149-1160. PMID: 19897823 
26. Mullen SP, Gothe NP, McAuley E. Evaluation of the Factor Structure of the Rosenberg Self-Esteem Scale in Older Adults. Pers Individ Dif. 2013;54(2):153-157. PMID: 23185099

27. Szcześniak M, Bielecka G, Madej D, Pieńkowska E, Rodzeń W. The role of self-esteem in the relationship between loneliness and life satisfaction in late adulthood: evidence from Poland. Psychol Res Behav Manag 2020; 13:1201-1212. PMID: 33363419.

28. Chan AC-M. Clinical validation of the Geriatric Depression Scale (GDS): Chinese Version. J Aging Health. 1996;8(2):238-253. PMID: 10160560.

29. Wang JJ. Prevalence and correlates of depressive symptoms in the elderly of rural communities in southern Taiwan. J Nurs Res. 2001;9(3):1-12. PMID: 12688568.

30. Cornwell EY, Waite LJ. Social disconnectedness, perceived isolation, and health among older adults. J Health Soc Behav. 2009;50(1):31-48. PMID: 19413133.

31. Chen YL, Hsiung PC, Chung L, Chen SC, Pan AW. Psychometric properties of the Mastery ScaleChinese version: applying classical test theory and Rasch analysis. Scand J Occup Ther. 2013;20(6):404-411. PMID: 24066856

32. Hsiung P-C, Pan A-W, Liu S-K, Chen S-C, Peng S-Y, Chung L. Mastery and stigma in predicting the subjective quality of life of patients with schizophrenia in Taiwan. J Nerv Ment Dis. 2010;198(7):494500. PMID: 00005053-201007000-00005.

33. Atkinson JW. Motivational determinants of risk-taking behavior. Psychol Rev. 1957;64, Part 1(6):359372. doi: $10.1037 / \mathrm{h} 0043445$.

34. Michou A, Matos L, Gargurevich R, Gumus B, Herrera D. Building on the enriched hierarchical model of achievement motivation: autonomous and controlling reasons underlying mastery goals. Psychol Belg. 2016;56(3):269-287 PMID: 30479440.

35. Liang K-Y, Zeger SL. Longitudinal data analysis using generalized linear models. Biometrika. 1986;73(1):13-22. doi: 10.1093/biomet/73.1.13

36. Wood CJ, Pretty J, Griffin M. A case-control study of the health and well-being benefits of allotment gardening. J Public Health. 2016;38(3):e336-e344. PMID: 26515229.

37. Lin CX, Lee C, Lally D, Coughlin JF. Human Aspects of IT for the Aged Population. Applications in Health, Assistance, and Entertainment. In: Zhou J., Salvendy G. (eds). Impact of virtual reality (VR) experience on older adults' well-being. Springer, Cham; 2018:89-100. doi: 10.1007/978-3-319-92037$5 \_8$

38. Padilha JM, Machado PP, Ribeiro A, Ramos J, Costa P. Clinical virtual simulation in nursing education: randomized controlled rrial. J Med Internet Res. 2019;21(3):e11529. PMID: 30882355.

39. Joshi S, Mooney SJ, Kennedy GJ, Benjamin EO, Ompad D, Rundle AG, et al. Beyond METs: types of physical activity and depression among older adults. Age and Ageing. 2016;45(1):103-109. doi: 10.1093/ageing/afv164.

40. Taiwan Association Against Depression. http://www.depression.org.tw/detection/index_06.asp. [Accessed 12th August, 2021]. 
41. Raeifar E, Halkett A, Lohman MC, Sirey JA. The relation between mastery, anticipated stigma and depression among older adults in a primary care setting. J Nerv Ment Dis. 2017;205(10):801-804. PMID: 28961595.

42. Brown JA. An exploration of virtual reality use and application among older adult populations. Gerontol Geriatr Med. 2019;5:2333721419885287. PMID: 31723574.

\section{Figures}

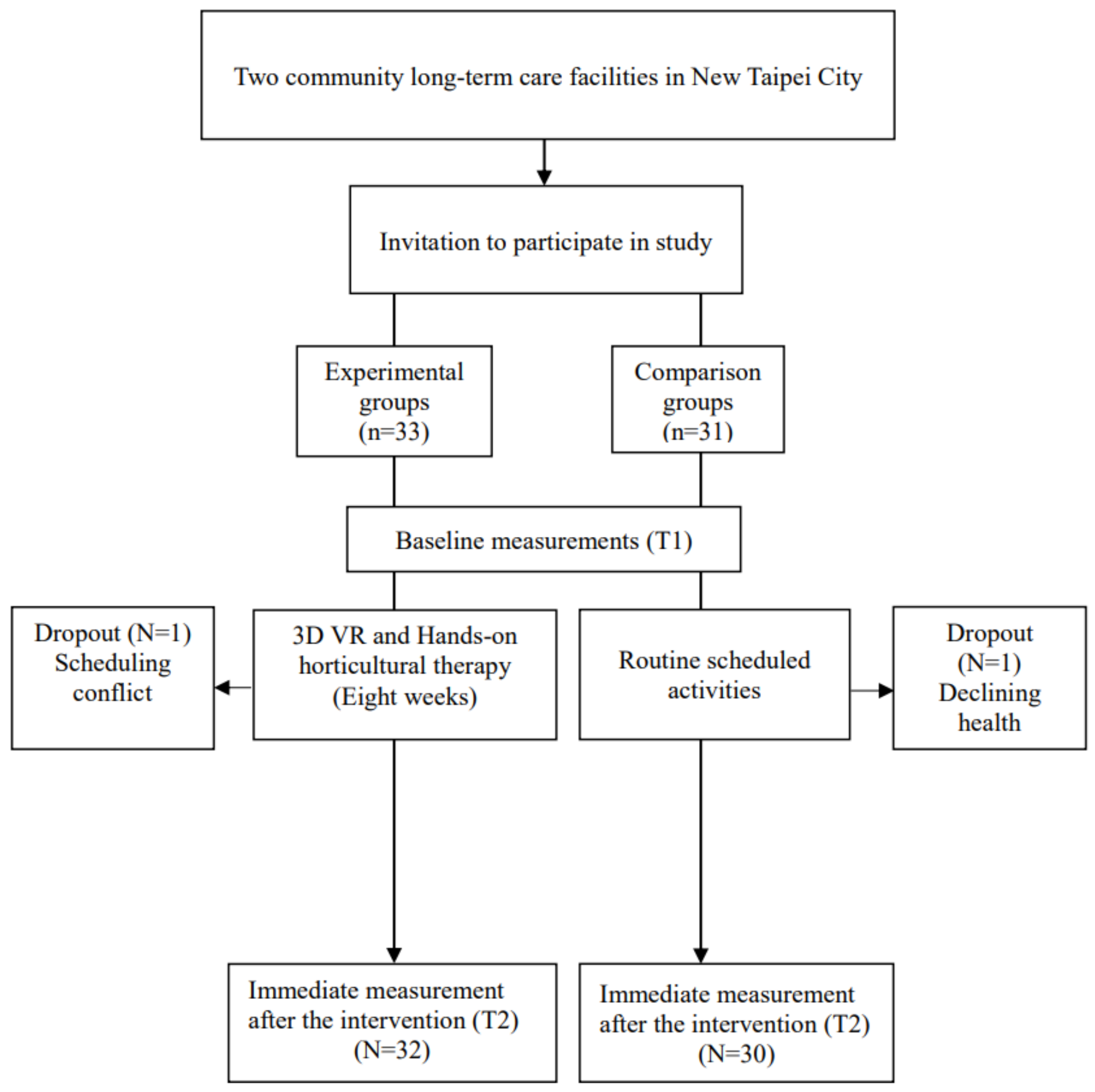


Figure 1

Enrollment and research process

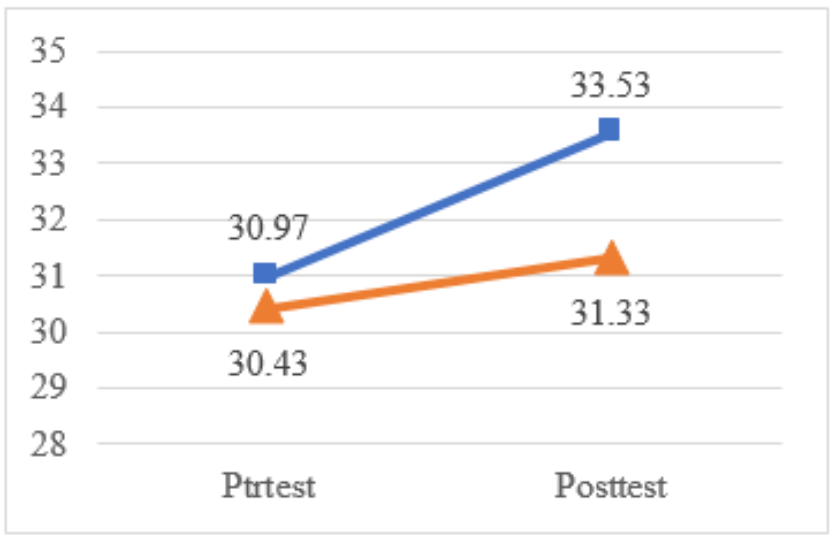

\section{(1) Self-esteem}

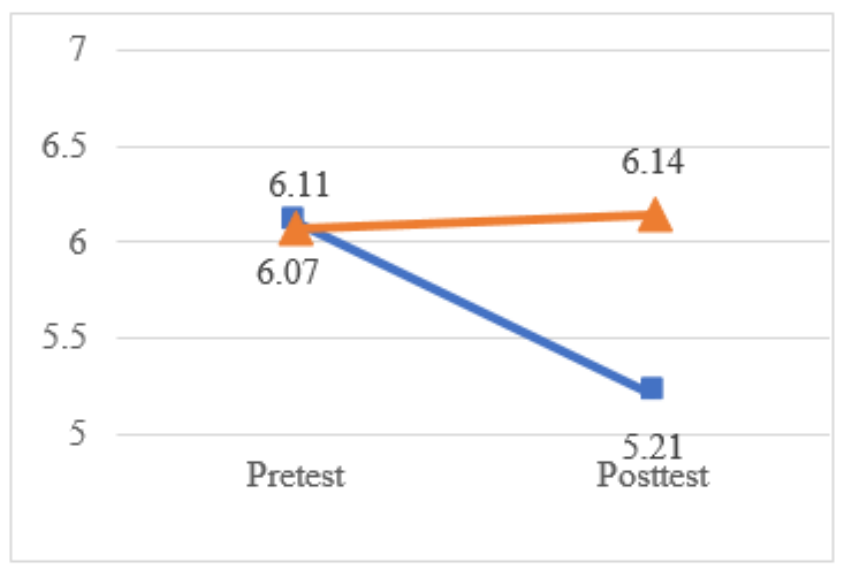

(3) Isolation

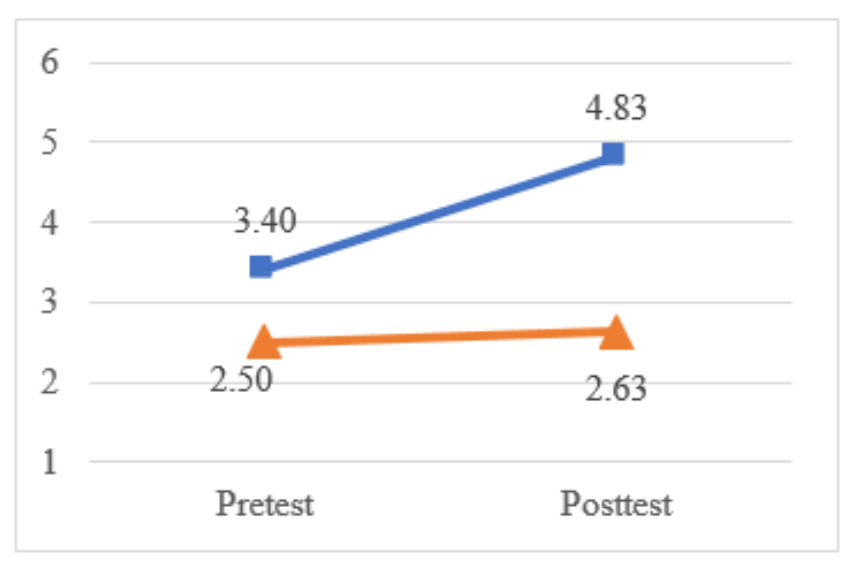

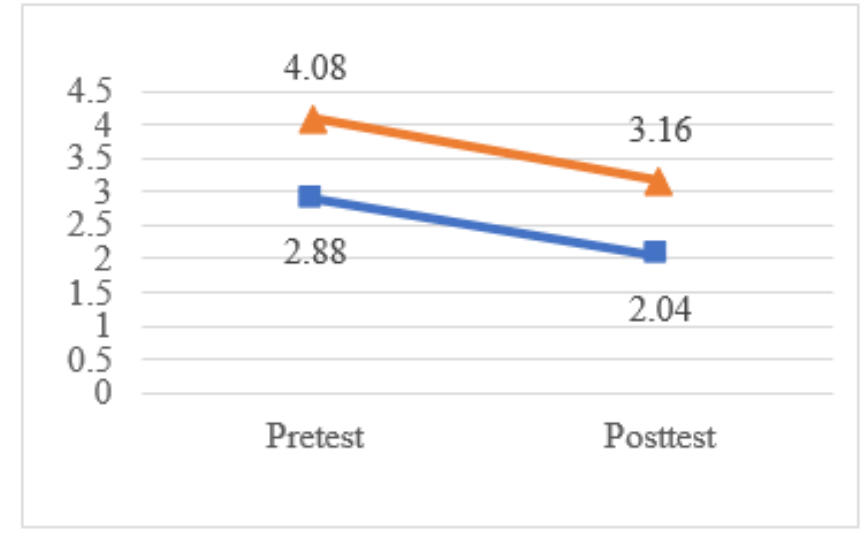

\section{(2) Depression}

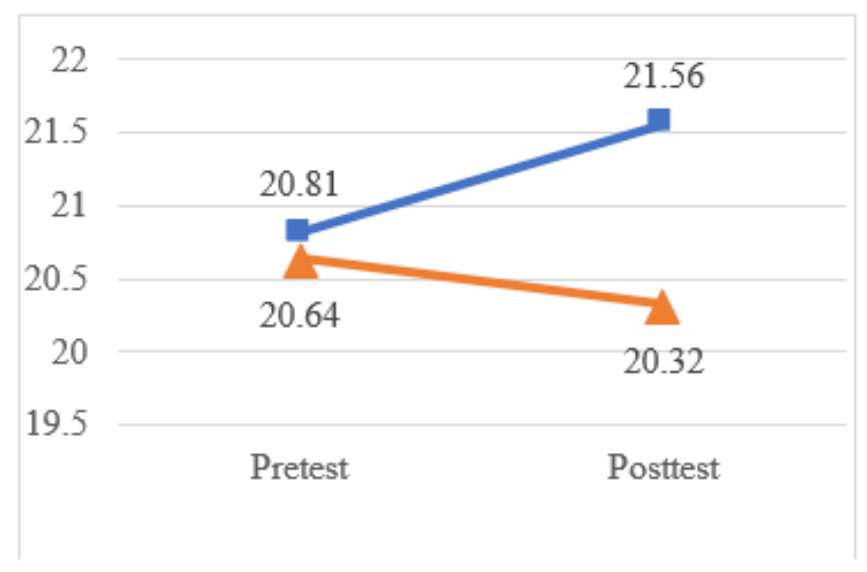

(4) Mastery

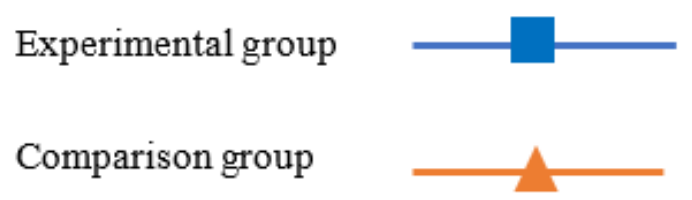

(5) Achievement Motives

\section{Figure 2}

Changes in pretests and posttests for five outcome variables between experimental and comparison groups. 